\title{
Maximality Principles in the Hyperuniverse Programme
}

\section{Sy-David Friedman \& Claudio Ternullo}

\section{Foundations of Science}

The official Journal of the Association for Foundations of Science, Language and Cognition

ISSN 1233-1821

Found Sci

DOI 10.1007/s10699-020-09707-8 
Your article is protected by copyright and all rights are held exclusively by Springer Nature B.V.. This e-offprint is for personal use only and shall not be self-archived in electronic repositories. If you wish to self-archive your article, please use the accepted manuscript version for posting on your own website. You may further deposit the accepted manuscript version in any repository, provided it is only made publicly available $\mathbf{1 2}$ months after official publication or later and provided acknowledgement is given to the original source of publication and a link is inserted to the published article on Springer's website. The link must be accompanied by the following text: "The final publication is available at link.springer.com". 


\title{
Maximality Principles in the Hyperuniverse Programme
}

\author{
Sy-David Friedman ${ }^{1}\left(\mathbb{D} \cdot\right.$ Claudio Ternullo $^{2}$ (])
}

Accepted: 27 September 2020

(c) Springer Nature B.V. 2020

\begin{abstract}
In recent years, one of the main thrusts of set-theoretic research has been the investigation of maximality principles for $V$, the universe of sets. The Hyperuniverse Programme (HP) has formulated several maximality principles, which express the maximality of $V$ both in height and width. The paper provides an overview of the principles which have been investigated so far in the programme, as well as of the logical and model-theoretic tools which are needed to formulate them mathematically, and also briefly shows how optimal principles, among those available, may be selected in a justifiable way.
\end{abstract}

Keywords Set theory $\cdot$ Universe of sets $\cdot$ Maximality principles $\cdot$ Hyperuniverse $\cdot$ New axioms

\section{The Question of Maximality}

One of the main goals of current set-theoretic research is to identify and justify maximality principles for the universe of sets $V .{ }^{1}$ Although the standard axioms of set theory, that is, ZFC, provide a nice characterisation of all sets as elements of $V$, they are not able to establish the truth or falsity of fundamental set-theoretic statements. Therefore, one may legitimately expect that isolating further fundamental properties of $V$ will provide us with crucial insights concerning these statements.

1 As is known, $V$ is recursively defined as follows: $V_{0}=\emptyset, V_{\alpha+1}=\mathcal{P}\left(V_{\alpha}\right)$, where $\alpha$ is a successor ordinal, and $V_{\lambda}=\bigcup_{\alpha<\lambda} V_{\alpha}$, where $\lambda$ is a limit ordinal.

The author would like to thank the FWF (Austrian Science Fund) for its support through the 'Hyperuniverse Project' (Grant P-28420).

The author wishes to thank the AGAUR for its support through the Beatriu de Pinós Post-Doctoral Fellowship 2018 BP 00192: 'Mathematical and Philosophical Aspects of a Multiversist Foundation of Set Theory'.

Claudio Ternullo

claudio.ternullo@ub.edu

1 Kurt Gödel Research Center for Mathematical Logic, University of Vienna, Kolingasse, 14-16, Wien 1090, Austria

2 Department of Mathematics and Computer Science, Universitat de Barcelona, Gran Via de les Corts Catalanes, 585, 08007 Barcelona, Spain 
In recent years, following Gödel's suggestion that maximality may be the key to a more thorough understanding of set-theoretic phenomena, set-theorists have focussed their attention on, among others, maximality principles. ${ }^{2}$ Informally, maximality principles prescribe that $V$ is a very rich structure, in particular the richest possible allowed by ZFC. At a more technical level, addressing the maximality of $V$ entails taking into account two features of the universe, that is, its height and its width. The height of the universe is expressed by the length of the sequence of ordinals indexing levels of $V$, whereas the width is expressed by the extent of the power-set operation, that is, by how many subsets of $V_{\alpha}$, for all ordinals $\alpha$, are produced at each successor level $V_{\alpha+1}$.

As a consequence, the maximality of $V$ can aptly be viewed as consisting of the following forms of maximality:

Height Maximality $V$ should be as high as possible: there should be as many as possible ordinals indexing levels of $V$

Width Maximality $V$ should be as wide as possible: as many as possible subsets should be produced at successor levels

As far as the former is concerned, in the last few decades, forms of the Reflection Principle (RP) have gradually stood out as the most attractive candidates as the 'ultimate' maximality principles expressing the height maximality of $V$. Intuitively, RP can be described as asserting that the universe cannot be uniquely characterised by any given collection of first-order properties. As is often said, the universe is indescribable. ${ }^{3}$ Strengthenings of first-order reflection, in particular, second-order reflection (with second-order parameters), are able to prove that there exist 'many' large cardinals, such as inaccessibles and Mahlos (in fact, proper classes of them), whose existence cannot be proved in ZFC. However, there are some limitative results which prevent RP from being extended 'too much'. ${ }^{4}$ As a consequence, one may legitimately ask: what, if any, is the maximal strengthening of RP, and how does one carry out such a strengthening mathematically? In recent years, the Hyperuniverse Programme (HP) has taken up the challenge of tackling these questions, by introducing a very strong reflection principle which embodies the ideal of a 'maximal strengthening' of RP (Sect. 4).

As far as width maximality is concerned, it does not seem plausible that something like RP may be extended also to the width of the universe, although attempts in this direction have been undertaken. As we shall see, HP follows a somewhat different strategy, by introducing a maximality principle which maximises the width of $V$ by maximising its inner models (Sect. 5).

Now, HP commits itself to delivering maximality principles for the height and the width of $V$, but the language in which such principles are couched implies the availability of

\footnotetext{
${ }^{2}$ For instance, see Gödel's oft-quoted remark in Gödel (1947), pp. 478-479: ‘..I am thinking of an axiom which (similar to Hilbert's completeness axiom in geometry) would state some maximum property of the system of all sets [...]. Note that only a maximum property would seem to harmonize with the concept of set [...]'. For a review of maximality principles in set theory, see Incurvati (2017).

3 At least, this is the form in which the principle can be stated if one subscribes to actualism (for which see Sect. 3 of this paper), a view that some scholars view as originating from Cantor's conception of a maximal, inextensible absolute infinite (for which see, in particular, Cantor's 1899 letter to Dedekind, in Ewald (1996), pp. 931-935), but the introduction of RP's may also be justified using a potentialist conception of $V$ (for which see, again, our section 3, or Tait (1998)). Incidentally, Gödel was also a major advocate of RP, to the point that he seems to have surmised that the axioms of set theory should be reduced to one single reflection axiom (see also Wang (1996), pp. 280-285, and Ternullo (2015), pp. 431-435).

4 A full account of such limitations is in Koellner (2009).
} 
'extensions' of $V$. As a consequence, two issues immediately arise: (1) the issue of what conception of $V$ is compatible with 'extensions'; (2) the issue of what logic is most suitable to express maximality principles which refer to 'extensions'. Both issues need careful addressing and, as we shall show, first-order logic will have to be strengthened to meet our purposes (Sect. 5.2). We will then be able to show that the programme's maximality principles not only have deep consequences in $V$ itself, but also in lower-level structures such as countable transitive models (whose collection we call the hyperuniverse) which turn out to be very useful for our approach (Sect. 5.3). The combination of height and width maximality principles, and the study of optimal versions thereof, will finally be taken into account (Sect. 6).

But before proceeding to review the mathematical work in full detail, in the next two sections, first (Sect. 2), we discuss what we call the 'maximality protocol', that is, the whole strategy behind HP's maximality programme, and discuss the use of such protocol to find optimal maximality principles. Afterwards (Sect. 3), we describe the conception of sets in terms of which the mathematical formulation of HP's maximality principles is best expressed.

Although also based on work expounded in other papers, ${ }^{5}$ the present paper is selfcontained, and represents the first comprehensive presentation of HP's 'maximality programme'.

\section{The Maximality Protocol}

Maximality in set theory (and in $V$ ) has been advocated and expressed in many ways, and within many different theoretical frameworks.

For instance, as is widely known, Maddy has devoted quite a substantial amount of work to showing that maximality, as encapsulated by her maxim MAXIMIZE, is one of the fundamental drives of set-theoretic research, leading, among other things, to the maximisation of the iterative process of 'construction' of $V$ itself. ${ }^{6}$ Moreover, on the 'MAXIMIZE view' of set theory, as Maddy is eager to show (with a view to providing arguments in favour of the acceptance of measurable, and other very large, cardinals), such axioms as $V=L$ will have to be rejected, as they restrict the range of possible 'isomorphism types' in $V$. $^{7}$

Authors such as Steel have presented an alternative way to express maximality, that is, as the maximisation of the 'interpretative power' of theories extending ZFC. In simple terms, given any two theories $T_{1}$ and $T_{2}$ extending ZFC by, respectively, adding two different (and sufficiently strong) large cardinal axioms, say, $\mathrm{LC}_{1}$ and $\mathrm{LC}_{2}$, ${ }^{8}$ whenever $T_{2}$ is consistency stronger than $T_{1}$, then the latter theory will be 'interpretable' in the other, that

\footnotetext{
5 See, in particular, Arrigoni and Friedman (2013), Antos et al. (2015), Friedman (2016), Friedman and Honzik (2016), Ternullo and Friedman (2016) and Antos et al. (2020).

6 What has been for some time known as the '(maximal) iterative concept of set' is described by, among others, Wang (1974) and Boolos (1971). Further discussion is in Parsons (1983) and Maddy (1988).

7 More specifically, Maddy's argument is that, since $V=L$ is inconsistent with the existence of $0^{\#}$, then under $\mathrm{ZFC}+V=L$, there will be fewer isomorphism types in $V$. See Maddy, Maddy (1997), pp. 219-232. Recently, the alleged 'restrictiveness' of $V=L$ has been challenged by Hamkins (2014), and further discussed by Incurvati and Löwe (2016).

${ }^{8}$ Or any two other theories whose consistency strength is, at least, that of ZFC+LC's.
} 
is, the set of all sentences provable in $T_{1}$ will be a subset of the set of all sentences provable in $T_{2} .{ }^{9}$ This, in turn, will yield a (consistency-strength-induced) linear hierarchy of theories each extending the interpretative power of the previous ones. Thus, the whole hierarchy of large cardinals clearly maximises over the 'interpretative power' of theories. ${ }^{10}$

Other set-theorists, instead, have placed more emphasis on the maximising virtues of such axioms as forcing axioms, which yield absoluteness (itself, a form of maximality) across structures (the set-generic multiverse) which are of common use in many set-theoretic investigations. ${ }^{11}$

All of these approaches share one fundamental feature: they construe maximality with reference to, and in light of, specific theories. But another approach is possible, one which focuses on the very properties of the cumulative hierarchy (of $V$ ), which, in turn, originate from the aforementioned iterative concept of set. On this approach, the properties to be maximised do not relate to theories, but rather to intuitive aspects of the hierarchy of sets.

As we have already hinted at in Sect. 1, by referring to the height and width of $V$, the bottom-up process of construction of the hierarchy of all sets can be seen as consisting in iterating the power-set and adding (ordinal-indexed) levels (or, what is the same in ZFC, adding ordinals). Thus, by keeping this fundamental insight about $V$, the main items of $V$ our principles will have to maximise are, precisely, power-sets and ordinals.

Now, one could also require that, along with these, one also maximises the cardinals. We may, for instance, want that, given a certain cardinal $\kappa$ in $V$, its successor, $\kappa^{+}$, is as big as possible. Of course, if one has maximised the ordinals then also the cardinals will, inevitably, turn out to be maximal, so cardinal maximality might just be seen as an intermediate step between ordinal and power-set maximality. However, as we will point out later on (Sect. 6.1), the maximisation of the power-set implies taking into account outer models which might not preserve the cardinals, so we will have to formulate ways to express cardinal maximality in such models.

To sum up, the whole process of maximisation may be summarised as a single 'maximality protocol' as follows:

(1) maximise the ordinals

(2) maximise the cardinals

(3) maximise the power-set

But this is not all there is to maximality on this approach, as one may also want to search for principles which maximise over more than just one of these three items (for instance, principles which are able to maximise over both the ordinals and the power-set). Ultimately, one might also want to find a single, optimal principle which maximises over all the items above. So, the maximality protocol above may be extended to the following, more general, one:

(1) Maximise (a.) the ordinals, (b.) the cardinals and (c.) the power-set

(2) Search for syntheses of principles maximising over, at least, two, among (a., b., c.)

\footnotetext{
${ }^{9}$ Steel (2014), p. 159. But notice: this is true only up to the level of second-order arithmetic, that is, up to the level of $\Pi_{\omega}^{1}$ sentences.

10 See Steel (2014), pp. 158-160. A theory $T$ is consistency stronger than a theory $U$ if $T$ proves $C o n(U)$. It should be noted that the theories addressed by Steel need to have at least the same consistency strength as that of ZFC+'there are infinitely many Woodin cardinals'.

${ }^{11}$ On this, see Bagaria (2005), and Woodin (2001).
} 
(3) Search for a single, optimal, maximality principle maximising over all of (a., b., c.)

In the last few years, the bulk of HP's research has been devoted to implementing the extended protocol above, and part of the job has also consisted in ascertaining whether a synthesis of, at least, any two among (a., b., c.) is consistent (something which one should not take for granted).

Now, when working on the 'maximality protocol', lots of maximality principles may be available for one to examine. Therefore, an essential aspect of this maximality programme will consist in being able to justifiably select principles which express some maximal property (for instance, ordinal maximality) in slightly different ways. It is sometimes hard to decide among different principles based on purely intuitive grounds. Our task may be facilitated by defining certain further, practical, criteria, such as the ones below, which, as will be clear later on, emerge very naturally in the context of HP's research:

Synthetic Features The first criterion looks very plausible and justified on the grounds of the extended maximality protocol: principles which maximise over more than one among (a., b., c.) in the maximality protocol, or which are more easily 'amenable' to a synthesis with other principles, are preferable over others which are not.

Inclusion of Parameters The use of parameters in a maximality principle just looks like a technical fact, which has nothing to do with the strength (let alone with the intuitive plausibility) of a maximality principle. However, as we shall see, producing parameterised versions of maximality principles (or of any other set-theoretic principle, for that matter) allows set-theorists to convey more information about properties of sets in $V$ which they may want to preserve. In particular, this strategy will be fundamental for our purpose of preserving cardinal maximality in maximality principles expressing power-set maximality. Thus, we stipulate that a maximality principle whose formulation also incorporates (certain) parameters is preferable over principles which do not contain (or contain fewer) parameters.

Nice Consequences This criterion partly corresponds to what is commonly known as an 'extrinsic argument' for the justification of an axiom (principle), ${ }^{12}$ that is, the fact that such an axiom (principle) has many (and far-reaching) desirable consequences (for instance, it decides $\mathrm{CH}$ ). Therefore, we stipulate that a axiom (principle) which has nice consequences is preferable over one which hasn't.

In Sect. 6.2 we will briefly show how the combination of these criteria may lead us to isolate HP's optimal maximality principles.

\section{Zermelo's Conception of Sets and Width Actualism}

As we have briefly hinted at, the question of how high the universe is, re-translates into the question of how long the ordinal sequence is, and the existence of different forms of RP, of different strength, which produce lengthenings of the sequence of ordinals, clearly shows that there is no immediate and trivial answer to this question.

On the other hand, the question of how wide the universe is looks a bit odd. After all, we seem to have a conception of the power-set operation, which should be able to provide us

\footnotetext{
12 For the terminology and its meaning, see Gödel (1947) and Gödel (1964). For a discussion of the relevance of 'extrinsic' arguments, see Maddy (1996).
} 
with an automatic indication of the width of the universe. However, the existence of inner models and forcing extensions of $V$ makes us immediately realise that this is not the case either. In particular, we know that such techniques as forcing, if taken at face value, seem to allow us to add 'new' subsets to $V$.

As a consequence, $V$ would seem to be 'extendible' somehow. But this isn't inevitable. One may, on the contrary, think that $V$ has to be construed as a fully determinate object, which means that no 'real' extensions of it may possibly be generated.

Therefore, two main ontological attitudes to $V$ are possible:

Potentialism $V$ is both 'extendible' in height and width.

Actualism $V$ is not extendible: its height and width are fully determinate.

As is clear, both attitudes are problematic: for instance, the potentialist needs explain how it is possible to conceive of literally adding new sets to $V$, if $V$ contains all of them. On the other hand, the actualist owes us an account of how, on her conception, one can make sense of extensions of the universe, such as those yielded by RP's, or of models such as forcing extensions $V[G]$ of $V$.

It should be noted that the meaning of 'maximality' and the justifiability of maximality principles varies significantly according to which of the two attitudes one adopts. ${ }^{13}$ An actualist will not be able to make sense of a literal construal of the idea of 'maximising $V$ ', insofar as she takes the universe to be already maximal. The potentialist, on the other hand, may also be at a loss with this idea, insofar as a straightforward consequence of his attitude is that there is no limit to the iterated production of new sets.

We will not provide a general examination of this complex issue here. Our aim is just to tackle the issue in connection with our need to express our maximality principles, for which we will adopt a mixed position which we may call width actualism, that is, the view that the cumulative hierarchy is a height-extendible object, whose width, on the contrary, cannot be extended.

Since, historically, this position was presented by Zermelo in his seminal Zermelo (1930) (with a view to showing that the axioms of set theory describe a fully determinate set-theoretic reality), in what follows we will interchangeably use width actualism and 'Zermelian conception'.

Zermelo first proved that, for a second-order version of the axioms, $\mathrm{ZFC}_{2}$, the power-set of $V$ is fixed. More precisely, he proved that any two models of $\mathrm{ZFC}_{2}$ are either isomorphic, or one is isomorphic to a proper initial segment of the other. As a consequence, the width of $V$ is fixed. ${ }^{14}$

Then, with regard to the height of $V$, Zermelo introduced the concept of a normal domain, a rank initial segment of $V$ indexed by what he called a limit-number which is a model of $\mathrm{ZFC}_{2}$. Now, the least such number must be the least strongly inaccessible cardinal. ${ }^{15}$ But then consider the theory $\mathrm{ZFC}_{2}+\exists \kappa$, where $\kappa$ is the least inaccessible. Such a theory is not true in $V_{\kappa}$, where $\kappa$ is the least inaccessible, so the least normal domain which satisfies $\mathrm{ZFC}_{2}+$ 'there is one inaccessible' must be $V_{\lambda}$, where $\lambda>\kappa$ is the least inaccessible after $\kappa$. The iterated procedure yields a 'tower' of nested models of $\mathrm{ZFC}_{2}$, and for any two

\footnotetext{
13 The topic is fully explored by one of the authors and Neil Barton in Barton and Friedman (2020).

14 Zermelo's proof is discussed (and defended) at length in Martin (2001). A philosophical discussion of Zermelo's conception is also in Tait (1998). Also see the more recent Linnebo (2017), pp. 179-182.

15 Recall that an uncountable cardinal $\kappa$ is inaccessible if and only if: (1) $\kappa$ is regular (that is, its cofinality is $\kappa$ ) and (2) $\kappa$ is a limit cardinal (that is, it is not the successor of any cardinal). $\kappa$ is strongly inaccessible if, in addition, is: (3) strong limit, that is, if for all cardinals $\lambda<\kappa, 2^{\lambda}<\kappa$.
} 
models $V$ and $V^{*}$ such that $V \subset V^{*}$, one has that the limit-number of $V^{*}$ is greater than the limit-number of $V$.

The collection of all V's may also be described as a vertical multiverse, although Zermelo's collection of stacked 'normal domains' is not, as is clear, a multiverse in the proper sense, nor is there any evidence that he construed the set-theoretic realm in a pluralist way: for him, set-theoretic reality was a fully determinate realm of objects, consisting of all sets in $V$; only, the concept 'universe of all sets' had, for him, an indefinite extension, insofar as what one might potentially take to be the collection of all sets at some level $V_{\kappa}$ (where $\kappa$ is, as said, at least inaccessible) can always automatically be extended to a larger collection $V_{\kappa+1}$, in which $V_{\kappa}$ is just a set. ${ }^{16}$ Among other things, this construction also allows one to make sense of higher-order quantification: given a normal domain indexed by $\kappa$, first-order variables will range over sets in $V_{K}$, and second-order variables over all subsets of $V_{\kappa}$ in $V_{\kappa+1}$.

Philosophically, it is not altogether clear that Zermelo's conception adequately assuages one's concerns about the referential indeterminacy of set-theoretic axioms. Certainly, for our more limited philosophical purposes, Zermelo's conception offers some other advantages, which can be summarised as follows:

(1) While this view provides us with a fairly rigid structural insight into $V$, it does not prevent talk of extensions (at least, height extensions)

(2) The view can motivate extensions of reflection to the next order very easily, and thus, motivate strong extensions of RP

(3) The view is able to justify the non-relativity of fundamental set-theoretic concepts, such as that of well-foundedness, which is central to our conception of $V^{17}$

However, Zermelo's conception has one major disadvantage: width extensions are not available in this picture of $V$. This fact has two major consequences: (1) set-theorists' intuition that first-order models are width-extendible is, on this account, unexplainable; (2) the maximisation of the power-set cannot be carried out, if not 'internally', as Zermelo's universe is already maximal, as far as its width is concerned.

Now, with regard to (2.), we will identify a way to express talk of width extensions within a non-width-extendible universe which allows one to make sense of width maximality principles, and, through this, we will also be able to address, if briefly, the more general philosophical issue expressed by (1.).

\footnotetext{
16 A different (and significantly more potentialist) construal of Zermelo's normal-domain construction may be found in Hellman (1989) and Linnebo (2013), both of which take end-extensions of $V$ to be interpretable in modal terms.

17 However, we do not claim the preferability of the second-order axioms of set theory. What we, rather, claim here is the view that the ordinary first-order iterative concept of set is constrained by (and even derivable from) the second-order concept, in such a way as to imply that the first-order understanding of the extendibility of $V$ should be seen as exclusively applying to the height of $V$ itself.
} 


\section{Height Maximality: Reflection}

Height maximality in terms of RP's for the universe $V$ can be intuitively formulated as follows: ${ }^{18}$

Reflection Any property which holds in $V$ already holds in some rank initial segment $V_{\alpha}$ of $V$.

In other words, $V$ cannot be described as the unique initial segment of the universe satisfying a given property. The strength of such reflection depends on what we take the word 'property' to mean. ${ }^{19}$ If this just means 'first-order property with set parameters' then we obtain Lévy reflection, a form of reflection provable in ZFC.

A priori, there is no need to limit ourselves to first-order properties of $V$. But to express second-order properties of $V$ we need to move beyond ZFC to Gödel-Bernays class theory GB. The latter has variables ranging over sets and also variables ranging over the larger collection of classes (collections of sets: note that every set is also a class). The $\in$-relation applies between sets and classes and we impose the Comprehension Scheme for formulas with only set-quantifiers (but with both set and class variables). Thus, in GB we can quantify over classes but cannot apply Comprehension to formulas containing such quantifiers. We also include Global Choice as an axiom, which says that there is a class function $F$ such that $F(x)$ is an element of $x$ for every nonempty set $x$.

GB is conservative over ZFC. However it can be strengthened by adding second-order reflection axioms to it, such as:

$\Pi_{m}^{1}$-Reflection If $\varphi(R)$ is a $\Pi_{m}^{1}$ formula with a class variable $R$, then reflection for $\varphi(R)$ is the implication

$$
\varphi(R) \rightarrow\left(V_{\alpha}, V_{\alpha+1}\right) \vDash \varphi\left(R \cap V_{\alpha}\right)
$$

where, on the right-hand-side, the set variables range over $V_{\alpha}$ and the class variables over $V_{\alpha+1}$.

Even $\Pi_{1}^{1}$-Reflection for sentences (without the class variable $R$ ) is rather strong, as it implies the existence of an inaccessible cardinal. That is because the regularity of an ordinal $\alpha$ is equivalent to the truth of a $\Pi_{1}^{1}$-sentence in $\left(V_{\alpha}, V_{\alpha+1}\right)$. By adding parameters, we get stronger large cardinals such as Mahlo cardinals and weakly compact cardinals.

But just as ZFC is inadequate for second-order reflection, GB is inadequate for thirdorder reflection. ${ }^{20}$ Of course, there is no reason to stop at third-order reflection, and in light

\footnotetext{
${ }^{18}$ See footnote 3 .

19 Properties are often formulated using higher-order quantification. Let $M$ be a class. We say that a variable $x$ is 1-st order (or of order 1) if it ranges over elements of $M$. In general, we say that a variable $R$ is $n+1$ -st order (or of order $n+1), 0<n<\omega$, if it ranges over $\mathcal{P}^{n}(M)$, where $\mathcal{P}^{n}(M)$ denotes the result of applying the powerset operation $n$ times to $M$. A formula $\varphi$ is $\Pi_{m}^{n}$ if it starts with a block of universal quantifiers of variables of order $n+1$, followed by existential quantification of variables of order $n+1$, and these blocks alternate at most $m-1$ times; the rest of the formula can contain variables of order at most $n+1$, and quantifications over variables of order at most $n . \Sigma_{m}^{n}$ is obtained by switching the words universal and existential.

${ }^{20}$ As an aside, it is worth noting that if formulated with third-order parameters, third-order reflection is in fact inconsistent! For instance, for a third-order parameter $\mathcal{R}$, i.e. a collection of classes, one is tempted by the following natural-looking principle:
}

Third-order Reflection If $\varphi(\mathcal{R})$ is true in $(V, \mathcal{R})$, then for some $\alpha, \varphi(\overline{\mathcal{R}})$ is true in $\left(V_{\alpha}, \overline{\mathcal{R}}\right)$, where $\overline{\mathcal{R}}=\left\{R \cap V_{\alpha} \mid R \in \mathcal{R}\right\}$.

But such a principle will fail if $\mathcal{R}$ consists of all bounded subsets of the ordinals (viewed as a collection of classes), and $\varphi(\mathcal{R})$ simply says that each element of $\mathcal{R}$ is bounded in the ordinals. Therefore when discussing third-order reflection it is customary to only allow second-order, and not third-order, parameters. 
of the Zermelian conception, it is meaningful to discuss ' $\alpha$-th order' reflection for ordinals $\alpha$ in lengthenings of $V$, i.e. in models $V^{*}$ which have $V$ as a rank initial segment.

This naturally leads to the following form of higher-order reflection:

Extended Reflection Axiom (ERA) $V$ satisfies the ERA if $V$ has a lengthening $V^{*}$, a model of ZFC, such that if $\varphi$ is first-order and $\varphi(A)$ holds in $V^{*}$, where $A$ is a subclass of $V$, then $\varphi\left(A \cap V_{\alpha}\right)$ holds in $V_{\beta}$ for some pair of ordinals $\alpha<\beta$ in $V$.

This allows us to reflect properties (with second-order parameters) that are $\alpha$-th order, for all ordinals $\alpha$ appearing in the least ZFC model lengthening of $V$. But, what is most important, the ERA embodies all of the classical forms of strong reflection and more.

However, clearly the ERA can easily be strengthened further, by requiring the lengthening $V^{*}$ of $V$ to satisfy more than ZFC, such as ZFC + 'there is a ZFC-lengthening of ZFC'. Indeed, it appears that there is no optimal form of reflection which can be described in terms of lengthenings of $V$, as we can always strengthen such a reflection principle further, by requiring a lengthening $V^{*}$ of $V$ in which the principle holds with reference only to lengthenings of $V$ appearing in $V^{*}$.

How are we, then, to achieve an optimal reflection principle? HP's answer is: through introducing the principle of \#-generation. This asserts the existence of a special kind of set called a \# (sharp) that 'generates' $V$ through iteration. An optimal RP results, since this iteration also produces a closed unbounded class of indiscernibles for $V$, adequate for witnessing any conceivable form of reflection. It is crucial that a \# which generates $V$ cannot be an element of $V$, otherwise such optimality would not be possible.

We cannot provide the full details of \#-generation here, but some of its essential features may be addressed. ${ }^{21}$

First, imagine that $V$ can be seen as being the last step in an elementary chain of universes $\left(V_{\kappa_{i}} \mid i<\infty\right)$ and we set $V=V_{\kappa_{\infty}}$. We can continue the construction of this chain 'beyond' $V$ itself, producing an upwards elementary chain of universes $V=V_{\kappa_{\infty}} \prec V_{\kappa_{\infty+1}} \prec V_{\kappa_{\infty+2}} \prec \cdots$.

By elementarity, all of these universes will satisfy the same first-order sentences, but we want more. We want that any two pairs of universes 'resemble' each other, i.e. satisfy the same first-order sentences, and this can be extended to any pair of $n$-tuples of universes $W_{\vec{i}}$, where $\vec{i}=i_{0}<i_{1}<\cdots<i_{n-1}$ and $W_{\vec{j}}$, where $\vec{j}=j_{0}<j_{1}<\cdots<j_{n-1}$ (to simplify our notation, we use the symbol $W_{i}$ for $V_{\kappa_{i}}^{*}$ ). But we want to impose an even higher level of resemblance, whereby all $n$-tuples of models satisfy the same second-order sentences and so on. In the end, the whole process can be seen as the construction of a series of embeddings $\pi_{i j}: V \rightarrow V$, leading to an indiscernibly-generated $V$. In more rigorous terms:

Definition 1 (Friedman, Honzik) $V$ is indiscernibly-generated iff: (1) There is a continuous sequence $\kappa_{0}<\kappa_{1}<\cdots$ of length $\infty$ such that $\kappa_{\infty}=\infty$ and there are commuting elementary embeddings $\pi_{i j}: V \rightarrow V$, where $\pi_{i j}$ has critical point $\kappa_{i}$ and sends $\kappa_{i}$ to $\kappa_{j}$. (2) For any $i \leq j$, any element of $V$ is first-order definable in $V$ from elements of the range of $\pi_{i j}$ together with $\kappa_{k}$ 's for $k$ in the interval $[i, j)$.

Indiscernible-generation has an equivalent but more useful formulation in terms of \# -generation, so we will use the term \#-generation for this strong form of reflection. Now, although adequate for the Zermelian conception, we shall see, in Sect. 6, that \#-generation

${ }^{21}$ See Section 2.2 of Friedman and Honzik (2016). 
needs to be slightly weakened, in order to fully suit width actualism, but this passage is far from being troublesome. ${ }^{22}$

One can show that \#-generation implies all forms of reflection which are compatible with $V=L,{ }^{23}$ and, as a consequence of this, we believe that \#-generation expresses the strongest possible amount of height reflection, and that it can legitimately claim to be the optimal principle expressing the height maximality of $V$. But why does it have to be so? Couldn't there be other (stronger) forms of reflection which imply $\neg(V=L)$, and which also account for the existence of much stronger large cardinal hypotheses?

Mathematically, research in this direction has been conducted, and has, in particular, produced what we will call here embedding reflection. ${ }^{24}$ Let $\overline{\mathcal{R}}$ result from applying the inverse of an elementary embedding to $\mathcal{R}$ in Third-Order Reflection (see, again, footnote 20). This very strong RP even yields supercompact cardinals (which are inconsistent with $V=L)$ ! However, the use of and reference to arbitrary embeddings makes this principle incompatible with our maximality protocol, which, as we have seen in Sect. 2, just addresses 'general' features of $V$, and not specific theories or model-theoretic techniques.

But there may be a more basic and cogent reason why any RP overtaking the $V=L$ barrier may not be seen as intuitively plausible: 'height maximality' refers to the length of the ordinal sequence and therefore if $V$ is height maximal, so is 'its' $L$, as $L$ has the same ordinals as $V$. It follows that any height maximality principle, compatible with a maximality protocol like ours, which only takes into account intuitive properties of $V$, must be consistent with $V=L$. As a consequence, the inevitable conclusion will be that any RP which contradicts $V=L$ may not be viewed as a genuine height maximality principle.

\section{Width Maximality: V-Logic, IMH}

\subsection{The Strategy}

As we have made clear already in Sect. 3, within the Zermelian conception, which incorporates height potentialism and width actualism, expressing principles of width maximality presents a real challenge. Whereas in the case of height maximality we made liberal use of lengthenings of $V$, no analogous notion of thickening (or outer model) of $V$ is available, on that conception.

Now, since Friedman (2006), the programme has expressed width maximality in terms of the following principle:

The Inner Model Hypothesis (IMH) If a first-order sentence holds in an inner model of some outer model of $V$ then it also holds in some definable inner model of $V$.

As is clear, the IMH is conceptually problematic for the Zermelian, as it explicitly refers to 'outer models' which are not available in Zermelo's picture of $V$. However, if the IMH were referring not to the whole $V$, but just to some countable transitive model (which we will mostly indicate as 'little- $V$ ') of ZFC, then the IMH would make perfect sense even within the Zermelian conception. ${ }^{25}$

\footnotetext{
22 See Sect. 5.3.2

23 The proof is in Friedman and Honzik (2016), p. 11.

24 See the discussion of this in Section 2.1 of Friedman and Honzik (2016), and also Welch (2019).

25 Note that IMH is also known to consistently hold for some choice of little- $V$. See Friedman et al. (2008).
} 
However, recent developments, first introduced in Antos et al. (2015), Friedman (2016), and then further examined in Antos et al. (2020), provide a solution to this problem. The introduction of $V$-logic enables one to express first-order properties of arbitrary outer models (almost) internally within $V$, in the same way as first-order properties of set-forcing extensions of $V$ can be internalised using the forcing relation. The word 'almost' occurs because this new 'truth in outer models' relation will not in general be first-order definable over $V$, but rather over a small lengthening (not thickening) of $V$ called $H y p(V)$ (the least 'admissible set' containing $V$ as an element). As lengthenings are available to the Zermelian, this enables her to express principles such as the IMH without loss of content.

Now, despite our motivated preference for the use of the Zermelian conception in HP, we shall also scrutinise a second approach to width maximality, which will construe the IMH as referring to some countable model 'little- $V$ ', whereas the first approach, the Zermelian conception, through the use of $V$-logic, only allows us to make sense of IMH 'as if' it were referring to the whole $V$. The reason for pursuing also the second approach is as follows. Although conceptually less justified, this approach is particularly convenient practically, as it allows us to reduce the study of the consequences of maximality principles to their consequences in countable transitive models.

\subsection{First Approach: V-Logic and IMH}

In order to discuss the first approach, we introduce the following analogy. As we said, the case of IMH is analogous to that of Martin's Axiom (MA), a principle of set-forcing. ${ }^{26}$ Several formulations of MA are available, in particular, $M A_{\aleph_{1}}$ asserts:

Outer-Model $M A_{\aleph_{1}}$. Whenever $V[G]$ is a generic extension of $V$ by a partial order $\mathbb{P}$ with the countable chain condition in $V$, and $\varphi(x)$ is a $\Sigma_{1}\left(\mathcal{P}\left(\omega_{1}\right)\right)$ formula (i.e. a $\Sigma_{1}$-formula with a subset of $\omega_{1}$ as parameter), if in $V[G]$ there is a $y$ such that $\varphi(y)$ holds, then there is also such a $y$ in $V$.

Note the quantification in this definition over the (generic) outer models $V[G]$ of $V$. How can the width actualist possibly make sense of this? The answer is of course via the definable forcing relation:

Internal $M A_{\aleph_{1}}$. Whenever $\mathbb{P}$ is a partial order with the countable chain condition in $V$, and $\varphi(x)$ is a $\Sigma_{1}\left(\mathcal{P}\left(\omega_{1}\right)\right)$ formula, if there is a forcing condition $p$ in $\mathbb{P}$ forcing the existence of a $y$ such that $\varphi(y)$ holds, then there is also such a $y$ in $V$.

These two formulations of $M A_{\aleph_{1}}$ are equivalent when $V$ is replaced by a countable transitive model 'little- $V$ ' of ZFC. When little- $V$ is not countable (and possibly equal to $V$ ), we use the latter internal formulation to express $M A_{\aleph_{1}}$. Thus, we convert a principle that makes reference to outer models of $V$ to one which is internal, expressible within $V$.

Now, in order to use the same strategy to formulate width maximality principles, we will use what has been called $V$-logic, and the whole point of this logic is precisely that it provides a tool to enable us to do the analogous thing not for just generic outer models, but for outer models in general.

$V$-logic has a symbol for $\in$, a predicate symbol $\bar{V}$ to denote $V$ and a constant symbol $\bar{x}$ to denote $x$ for each set $x$. The proof relation $\vdash_{V}$ of $V$-logic begins with axioms that assert that $\bar{x}$ belongs to $\bar{V}$ for each set $x$, together with the usual axioms of first-order logic and all

$\overline{{ }^{26}}$ For further on this analogy, see Barton and Friedman (2020). 
quantifier-free sentences true in $V$. The rules of inference are modus ponens together with the infinitary rules:

- From $\varphi(\bar{y})$ for all $y$ in $x$, infer $\forall y \in \bar{x} \varphi(y)$.

- From $\varphi(\bar{x})$ for all $x$ in $V$, infer $\forall x \in \bar{V} \varphi(x)$.

Proofs are then well-founded trees which can be shown to belong to $H y p(V)$, the least admissible set containing $V$ as an element. Assuming height potentialism (which is available to the Zermelian conception), $\operatorname{Hyp}(V)$ makes full sense. ${ }^{27}$

As said, now we proceed in a way fully analogous to what we did above using the forcing relation. Reconsider the IMH:

The Inner Model Hypothesis. If a first-order sentence holds in an inner model of some outer model of $V$ then it also holds in some definable inner model of $V$.

We then formulate an internal version of this as follows:

The Internal Inner Model Hypothesis. If the theory in $V$-logic $T_{\varphi}$ asserting that the firstorder sentence $\varphi$ holds in an inner model of some outer model of $\bar{V}$ is consistent in $V$-logic, then there is a definable inner model of $V$ in which $\varphi$ holds.

The 'internal' IMH is expressible as a first-order property of $\operatorname{Hyp}(V)$, using the fact that the consistency of $T_{\varphi}$ in $V$-logic is equivalent to saying that there is no $V$-logic proof in $\operatorname{Hyp}(V)$ of a contradiction using the axioms of $T_{\varphi}$. And as in the case of $M A_{\aleph_{1}}$, the two formulations of the IMH, the one using outer models and the internal one, are equivalent when $V$ is replaced by a countable transitive model little- $V$ of ZFC.

Thus, $V$-logic opens the way to expressing a wide range of width maximality principles in the Zermelian (width actualist) context. With rare exceptions, these principles are formalisable internally in $H y p(M)$ for arbitrary transitive ZFC models $M$, and not just for countable ones.

\subsection{Second Approach: Reduction to the Hyperuniverse}

\subsubsection{Reduction of IMH}

As promised, we shall now deal with the second approach, where $V$ is taken to be a countable transitive model little- $V$. Moreover, in this section we show that we can reduce our study of width maximality, and to some extent of height maximality, to a study of countable transitive models. As the collection of countable transitive models has been called 'hyperuniverse', we are led to reduce the study of the consequences of maximality principles to their consequences in the hyperuniverse.

First we illustrate the reduction to the hyperuniverse with the specific example of the IMH. Suppose that we formulate the IMH as above, using $V$-logic, and want to know what first-order consequences it has.

Fact 2 Suppose that a first-order sentence $\varphi$ holds in all countable models of the IMH. Then it holds in all models of the IMH.

${ }^{27} V$-logic is an extension (reformulation) of Barwise's $\mathfrak{M}$-logic, for which see Barwise (1975). 
This is for the following reason: suppose that $\varphi$ fails in some model $M$ of the IMH, where $M$ may be uncountable. Now notice that the IMH is first-order expressible in $\operatorname{Hyp}(M)$, the least admissible lengthening of $M$. But then, apply the downward LöwenheimSkolem theorem to obtain a countable little- $V$ which satisfies the IMH, as verified in its associated little-Hyp $(V)$, yet fails to satisfy $\varphi$. But this is a contradiction, as by hypothesis $\varphi$ must hold in all countable models of the IMH.

So without loss of generality, when looking at first-order consequences of width maximality principles as formulated in $V$-logic, we can restrict ourselves to countable little- $V$ 's. The advantage of this is that, then, we can dispense with little- $V$-logic, as by the Completeness Theorem for little- $V$-logic, consistent theories in little- $V$-logic do have models, thanks to the countability of little- $V .^{28}$ Thus, for a countable little- $V$, the IMH simply says:

Inner Model Hypothesis for little- $V$ 's. Suppose that a first-order sentence holds in an inner model of an outer model of little- $V$. Then it holds in a definable inner model of little- $V$.

But, if $V$ is taken to be little- $V$, then $V$ can really be 'thickened', which means that the Zermelian conception collapses to a fully potentialist conception, wherein both height and width of $V$ are not fixed. Alternatively said, the Zermelian and the fully potentialist versions of the IMH coincide on countable models.

\subsubsection{Reduction of \#-Generated V:\#-Generation Revisited}

As far as the case of \#-generation is concerned, its reduction to the hyperuniverse is not so obvious, and we shall see that the choice of working either within a Zermelian conception or within a fully potentialist perspective makes a big difference.

First, consider the following encouraging analogue for \#-generation of our earlier reduction claim for the IMH, which we state here without proof.

Fact 3 Suppose that a first-order sentence $\varphi$ holds in all countable models which are \# -generated. Then it holds in all models which are \#-generated.

Now, the main difficulty, we briefly touched on in $\S 4,{ }^{29}$ is this: how do we express \# -generation from a width actualist perspective? As explained in Sect. 4, to produce a generating \# for $V$ we have to produce a set of rank less than $\operatorname{Ord}(V)$ which does not belong to $V$. So, we now have to face one further difficulty for the width actualist, as far as height maximality is concerned and, at this point, we need to say a bit more about \#'s and models generated by them.

A pre-\# is a structure $(N, U)$ where $U$ measures the subsets in $N$ of the largest cardinal $\kappa$ of $N$, meeting certain first-order conditions; it is a \# if in addition it is iterable, i.e. for any ordinal $\alpha$, if we take iterated ultrapowers of $(N, U)$ for $\alpha$ steps, then, it remains wellfounded. $V$ is \#-generated if it results as the union of the lower parts of the $\alpha$-iterates of some \# as $\alpha$ ranges over $\operatorname{Ord}(V)$. But notice that to express the iterability of a generating \# for $V$ we are forced to consider theories $T_{\alpha}$ formulated in $L_{\alpha}(V)$-logic for arbitrary (Gödel-) lengthenings $L_{\alpha}(V)$ of $V: T_{\alpha}$ asserts that $V$ is generated by a pre-\# which is $\alpha$-iterable, i.e.

\footnotetext{
28 The completeness result for little- $V$-logic is equivalent to the completeness result for countable fragments of Barwise's $\mathfrak{M}$-logic. See Barwise (1975), p. 89.

29 See the paragraph immediately below Definition 1.
} 
iterable for $\alpha$-steps. Thus, we have no fixed theory that captures \#-generation, only a tower of theories $T_{\alpha}$ (as $\alpha$ ranges over ordinals past the height of $V$ ) which capture closer and closer approximations to \#-generation.

Therefore, in order to overcome these difficulties, we need to introduce another form of \#-generated $V$, that is, weakly \#-generated $V$.

Definition $4 V$ is weakly \#-generated if for each ordinal $\alpha$ past the height of $V$, the theory $T_{\alpha}$ which expresses the existence of an $\alpha$-iterable pre-\# which generates $V$ is consistent.

Weak \#-generation is meaningful for a width actualist who is also a height potentialist (that is, a Zermelian), as it is expressed entirely in terms of theories internal to lengthenings of $V$, and so weak \#-generation is precisely the form of height maximality which suits the Zermelian conception.

Now, a countable little- $V$ is weakly \#-generated if it is $\alpha$-generated for each countable ordinal $\alpha$ (where the witnessing pre-\# may depend on $\alpha$ ). Little- $V$ is \#-generated iff it is $\alpha$ -generated when $\alpha=\omega_{1}$ iff it is $\alpha$-generated for all ordinals $\alpha$. Finally, we have the following reduction to countable little- $V$ 's:

Fact 5 Suppose that a first-order sentence $\varphi$ holds in all countable little-V's which are weakly \#-generated, and this is provable in ZFC. Then $\varphi$ holds in all models which are weakly \#-generated.

To summarise: if we are fully potentialist, then we can comfortably work with full \# -generation as our principle of height maximality. But, if we are width actualists (Zermelians), we instead work with weak \#-generation, expressed in terms of theories inside Gödel lengthenings $L_{\alpha}(V)$ of $V$. Weak \#-generation is sufficient to maximise the height of the universe. And properly formulated, the reduction to the hyperuniverse also applies to weak \#-generation: to infer that a first-order statement follows from weak \#-generation, it suffices to show that in ZFC one can prove that it holds in all weakly \#-generated countable models. ${ }^{30}$

In what follows we will primarily work with \#-generation, as at present the mathematics of weak \#-generation is poorly understood. Indeed, as we shall see in the next section, a synthesis of \#-generation with the IMH is consistent, but this remains an open problem for weak \#-generation.

\section{Synthesis and Optimality}

Let's take stock. In Sects. 4 and 5, we have addressed (1a.) and (1c.) in the maximality protocol, that is, we have formulated maximality principles which maximise over the ordinals and the power-set. So far, we have not explicitly dealt with (1b.), cardinal maximality. The reason, as already noticed in Sect. 2, is that, once one 'fixes' the ordinals, then one also

\footnotetext{
30 Weak \#-generation is indeed strictly weaker than \#-generation for countable models. Suppose that $0^{\#}$ exists and choose $\alpha$ to be least, so that $\alpha$ is the $\alpha$-th Silver indiscernible ( $\alpha$ is countable). Now let $g$ be generic over $L$ for Lévy collapsing $\alpha$ to $\omega$. Then by Lévy absoluteness, $L_{\alpha}$ is weakly \#-generated in $L[g]$, but it cannot be \#-generated in $L[g]$ as $0^{\#}$ does not belong to a generic extension of $L$.
} 
maximises over the cardinals. However, there is another kind of cardinal maximality we will have to discuss here, which consists in 'fixing' the cardinals in outer models addressed by power-set maximality principles.

Afterwards, to fufil point (2.) and (3.) of the maximality protocol, we will proceed to examine syntheses of the maximality principles expressed in Sects. 4 and 5, which, as said, will also incorporate cardinal maximality. Moreover, in light of the reduction to the hyperuniverse $(\mathbb{H})$, we will see that maximality principles such as \#-generation and the IMH can, without loss of generality, be expressed as principles about countable models, i.e. as properties of members of $\mathbb{W}$ expressed through quantification over $\mathbb{H}$. Henceforward, we refer to them, and to their variants, as $\mathbb{H}$-principles.

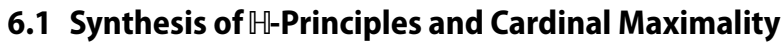

Arguably, the most important synthesis we may want to formulate is the synthesis of the $\mathbb{U}$ -principle of \#-generation, expressing height maximality, with $\mathbb{H}-$-principles which express width maximality. The first example of such a synthesis is the IMH\#, which asserts the IMH for height-maximal universes:

Definition 6 (IMH\#) $M$ satisfies the IMH\# if $M$ is \#-generated and whenever a first-order sentence holds in a \#-generated outer model of $M$, it also holds in a definable inner model of $M$.

IMH\# captures both height maximality and aspects of width maximality simultaneously. But the development of 'synthetic' $\mapsto$-principles does not stop here. At this point, one introduces further logical constraints, which allow one to also incorporate the kind of cardinal maximality described at the beginning of this section, as follows.

An absolute parameter is a set $p$ which is uniformly definable over all outer models of $V$ which 'respect' them in the sense that they preserve cardinals up to and including the cardinality of the transitive closure of $p$. The SIMH (Strong IMH) is the IMH for sentences with absolute parameters relative to outer models which respect them:

Definition 7 (SIMH) If a sentence with absolute parameters holds in an outer model which respects those parameters then it holds in a definable inner model.

Most important for our goal of incorporating cardinal maximality is the following definition:

Definition 8 (Cardinal Absoluteness) A cardinal-absolute parameter is a set $p$ which is uniformly definable over all cardinal-preserving extensions of $V$.

Now, CPIMH asserts the following:

Definition 9 (CPIMH) If a sentence with cardinal-absolute parameters holds in a cardinalpreserving outer model of $V$ it also holds in a definable inner model of $V$.

Restricting SIMH and CPIMH to \#-generated universes yields corresponding principles SIMH\# and CPIMH\#. 


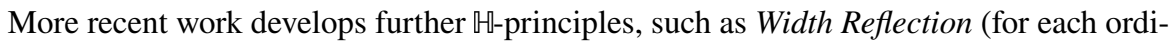
nal $\alpha$ there is an amenable elementary embedding of an inner model into $V$ with critical point greater than $\alpha$ ) and its associated analogue of \#-generation for width called Width Indiscernibility and Omniscience (the first-order definability of satisfaction across outer models), but all of these need further investigation to ultimately be viewed as plausible maximality principles. ${ }^{31}$

\subsection{Optimality}

We, finally, proceed to briefly address the issue of optimality, also based on the 'practical' criteria formulated in Sect. 2.

First, let us summarise all the work done so far, in view of HP's maximality programme. As far as point (1a.), the maximisation of the ordinals, is concerned, we may say that this is expressed by \#-generation, as discussed in Sect. 4, whereas point (1c.)., the maximisation of the power-set, is expressed by the IMH and its variants, in the way suggested in Sect. 5. Now, as far as point (1b.), cardinal maximality, is concerned, we have seen that the latter can be dealt with by fulfilling (1a.) and (1c.), and by incorporating cardinal parameters in versions of IMH and \#-generated $V$.

In order to select our optimal maximality principle, we proceed as follows. First, we choose the strongest principles, among those maximising over one item of the maximality protocol. So, we choose SIMH for power-set maximality and (strong) \#-generated $V$ for ordinal maximality, and produce SIMH\#. Finally, we also add cardinal-absolute parameters to SIMH\#, which leads to SIMH\#(CP). This principle contains all three forms of maximality. Now, is it justified on the grounds of the criteria we have put forward in Sect. 2? We believe so. SIMH\#(CP) is certainly synthetic, and, in addition, contains parameters. But SIMH\#(CP) also has nice consequences, as it has been shown that:

Fact 10 Under SIMH\#(CP), $\mathrm{CH}$ is false.

Therefore, we may finally state that SIMH\#(CP) should be seen, for the time being, and in the light of the justificatory strategy outlined in Sect. 2, as HP's optimal maximality principle.

There is one big problem with this principle, though, that is, the fact that we presently do not know whether it is consistent (although there are hints that it may be so), so, for now, we have to be very cautious about viewing it as the ultimate maximality principle for $V$.

\section{Concluding Remarks: The Significance of Maximality for the Foundations of Set Theory}

After having provided a comprehensive overview of HP's maximality programme, we want to briefly address one last philosophical issue relating to the significance of maximality principles for the whole set-theoretic undertaking.

One might wonder whether selecting 'optimal' maximality principles will imply that we will ultimately be able to also describe an 'optimal' picture of $V$. There are two

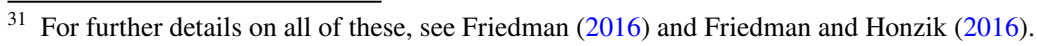


considerations to make concerning this point, but first we would like to set forth what alternative options are available for us to take into account concerning set-theoretic ontology.

Pluralism (or multiversism) is the view that there are multiple universes of sets, whereas universism (or monism), is the view that there is a single structure incorporating all the (unique) truths about sets. ${ }^{32}$ Now, we want to draw attention to the following fact. Even if we were able to single out some optimal principle $P$, it may still turn out that $P$ could be compatible with a wide range of universes, and, thus, compatible with a pluralist conception of set theory. ${ }^{33}$

Moreover, finding optimal maximality principles might not necessarily be seen as leading to a resolution of the monism/pluralism dichotomy for a different reason. Linnebo has recently come up with an instructive case, where a set-theoretic principle is judged to be sufficiently justified to be accepted as a new axiom. There may be two equally acceptable interpretations of this fact: either the 'discovery' of such an axiom was the result of settheorists' progressive sharpening of their grasp of $V$, something which would lend support to universism, or, on the contrary, it was the outcome of the progressive sharpening of their grasp of one (among many) set-theoretic structures (or of one among many 'concepts of set') something which would lend support to pluralism. Linnebo concludes:

So long as both interpretations are available, mathematical practice can proceed unaffected by the question of whether monism or pluralism is right. These reflections suggest that the question of pluralism matters less to mathematical practice than one might initially have thought. After all, monists and pluralists may agree that there is a plethora of mathematical structures that are worth exploring, [...]. Which of these structures corresponds to the 'real' sets may not be so important. (Linnebo 2017, p. 182)

It should be noted that the HP's construal of pluralism is slightly different from that suggested by Linnebo. In particular, the HP construes the relationship between 'concepts of set' and 'structures' as follows: there is just one concept of set, the maximal iterative concept, which is sufficient to justify and motivate the 'construction' of $V$, yet different 'pictures of $V$ ' (arising from 'extending' $V$ ) are available, which instantiate this concept. However, Linnebo's considerations also apply to the HP's picture, insofar as the identification of an optimal maximality principle $P$ may either be construed as arising from the progressive sharpening of the grasp of the unique concept of set (and unique universe satisfying it), or from the progressive sharpening of the grasp of one structure among all those satisfying this concept and $P$.

\footnotetext{
32 It should be noted that, although the hyperuniverse, introduced in Sect. 5.3, may be construed as a collection of mutually alternative 'universes' of set theory, and may thus qualify as a multiverse, in this paper we do not automatically subscribe to, or wish to defend, a multiversist view, since, as also explained in the present section, several ontological conceptions are compatible with HP's maximality protocol. In fact, as stated in Sect. 5, the 'reduction to the hyperuniverse' can just be seen as a convenient way to address the expression of maximality principles semantically, rather than as a way of advocating the actual existence of a collection of mutually alternative universes of set theory. We thank one anonymous referee for prompting us to clarify this point.

${ }^{33}$ For instance, take $P$ to be the combination of a well-known axiom such as $V=L$ and \#-generation. Suppose, however implausible it may seem, that one chose $P$ as the ultimate, optimal maximality principle. One would, then, realise that such a principle would still be compatible with many universes of the form $L_{\alpha}$, where $\alpha$ is a limit of Silver indiscernibles. Notice, though, that the identification of $P$ as an optimal maximality principle would, at least, narrow the range of universes of interest in the multiverse, as presumably only some universes will satisfy it.
} 
To sum up, the ontological interpretation of the discovery of optimal principles may not be so straightforward. This fact, in turn, may not have too much significance for the search for the ultimate maximality principles: overall, optimality expresses epistemological, rather than ontological concerns, which means that a principle may be chosen (and adopted) not because it corresponds to, or provides us with some nice and unified picture of, set-theoretic ontology, but because it best encapsulates the relevant features of the concept of set, as standardly interpreted.

We feel optimistic in this respect: further 'experimenting' with maximality principles, and with their properties, within the HP, may give us optimal maximality principles which will also be seen as capable of settling many of the most important open problems in set theory.

\section{Compliance with Ethical Standards}

Conflict of interest On behalf of all authors, the corresponding author states that there is no conflict of interest.

\section{References}

Antos, C., Barton, N., \& Friedman, S.-D. (2020). Universism and Extensions of V. Review of Symbolic Logic, forthcoming.

Antos, C., Friedman, S.-D., Honzik, R., \& Ternullo, C. (2015). Multiverse conceptions in set theory. Synthese, 192(8), 2463-2488.

Arrigoni, T., \& Friedman, S. (2013). The hyperuniverse program. Bulletin of Symbolic Logic, 19(1), 77-96.

Bagaria, J. (2005). Natural axioms of set theory and the continuum problem. In: Hájek, P., Valdés-Villanueva, L., and Westertåhl, D., (eds.,) Logic, Methodology and Philosophy of Science. In: Proceedings of the 12th International Congress, pages 43-64. King's College Publications, London.

Barton, N., \& Friedman, S.-D. (2020). Maximality and Ontology: How axiom content varies across philosophical frameworks. Synthese, 197, 623-649.

Barwise, J. (1975). Admissible Sets and Structures. Berlin: Springer.

Boolos, G. (1971). The iterative conception of set. Journal of Philosophy, 68(8), 215-231.

Ewald, W. (1996). From Kant to Hilbert: A Source Book in the Foundations of Mathematics. Oxford: Oxford University Press.

Friedman, S. (2006). Internal consistency and the inner model hypothesis. Bulletin of Symbolic Logic, 12(4), 591-600.

Friedman, S. (2016). Evidence for set-theoretic truth and the hyperuniverse programme. IfCoLog Journal of Logics and their Applications, 3(4), 517-555.

Friedman, S., \& Honzik, R. (2016). On strong forms of reflection in set theory. Mathematical Logic Quarterly, 62(1-2), 52-58.

Friedman, S., Welch, P., \& Woodin, W. H. (2008). On the consistency strength of the inner model hypothesis. Journal of Symbolic Logic, 73(2), 391-400.

Gödel, K. (1947). What is Cantor's continuum problem? American Mathematical Monthly, 54, $515-525$.

Gödel, K. (1964). What is Cantor's continuum problem? In P. Benacerraf \& H. Putnam (Eds.), Philosophy of Mathematics Selected Readings (pp. 470-85). New Jersey: Prentice-Hall.

Hamkins, J. D. (2014). A multiverse perspective on the axiom of constructiblity. In Infinity and Truth, volume 25 of Lect. Notes Ser. Inst. Math. Sci. Natl. Univ. Singap., pages 25-45. World Sci. Publ., Hackensack, NJ.

Hellman, G. (1989). Mathematics without Numbers Towards. A Modal-Structural Interpretation. Oxford: Clarendon Press.

Incurvati, L. (2017). Maximality principles in set theory. Philosophia Mathematica, 25(2), 159-193.

Incurvati, L., \& Löwe, B. (2016). Restrictiveness relative to notions of interpretation. Review of Symbolic Logic, 9(2), 238-50.

Koellner, P. (2009). On reflection principles. Annals of Pure and Applied Logic, 157(2-3), 206-19.

Linnebo, Ø. (2013). The potential hierarchy of sets. Review of Symbolic Logic, 6(2), 205-228. 
Linnebo, Ø. (2017). Philosophy of Mathematics. Princeton: Princeton University Press.

Maddy, P. (1988). Believing the axioms, I. Bulletin of Symbolic Logic, 53(2), 481-511.

Maddy, P. (1996). Set-theoretic naturalism. Bulletin of Symbolic Logic, 61(2), 490-514.

Maddy, P. (1997). Naturalism in Mathematics. Oxford: Oxford University Press.

Martin, D. (2001). Multiple universes of sets and indeterminate truth values. Topoi, 20, 5-16.

Parsons, C. (1983). What is the iterative conception of sets? In P. Benacerraf \& H. Putnam (Eds.), Philosophy of Mathematics Selected Readings (pp. 503-529). Cambridge: Cambridge University Press.

Steel, J. (2014). Gödel's program. In J. Kennedy (Ed.), Interpreting Gödel Critical Essays (pp. 153-179). Cambridge: Cambridge University Press.

Tait, W. W. (1998). Zermelo's conception of set theory and reflection principles. In M. Schirn (Ed.), Philosophy of Mathematics Today (pp. 469-483). Oxford: Clarendon Press.

Ternullo, C. (2015). Gödel's cantorianism. In G. Crocco \& E.-M. Engelen (Eds.), Kurt Gödel: PhilosopherScientist (pp. 413-442). Aix-en-Provence: Presses Universitaires de Provence.

Ternullo, C., \& Friedman, S.-D. (2016). The search for new axioms in the hyperuniverse programme. In F. Boccuni \& A. Sereni (Eds.), Philosophy of Mathematics: Objectivity, Realism and Proof Filmat Studies in the Philosophy of Mathematics, Boston Studies in Philosophy of Science (pp. 165-188). Berlin: Springer.

Wang, H. (1974). From Mathematics to Philosophy. London: Routledge \& Kegan Paul.

Wang, H. (1996). A Logical Journey. Cambridge (MA): MIT Press.

Welch, P. (2019). Proving theorems from reflection. In S. Centrone, D. Kant, \& D. Sarikaya (Eds.), Reflections on the Foundations of Mathematics: Univalent Foundations, Set Theory, and General Thoughts (pp. 79-97). Berlin: Springer.

Woodin, W. H. (2001). The Continuum Hypothesis. Notices of the American Mathematical Society, Part 1: 48, 6, p. 567-76; Part 2: 48, 7, p. 681-90.

Zermelo, E. (1930). Über Grenzzahlen und Mengenbereiche: neue Untersuchungen über die Grundlagen der Mengenlehre. Fundamenta Mathematicae, 16, 29-47.

Publisher's Note Springer Nature remains neutral with regard to jurisdictional claims in published maps and institutional affiliations.

Sy-David Friedman earned his PhD in Mathematical Logic at MIT in 1976. After serving on the MIT faculty until 2004, he founded the Kurt Gödel Research Center for Mathematical Logic at the University of Vienna, which continues to be a world-leading center for research in both Mathematical Logic and the Foundations of Mathematics. Friedman has published more than 180 articles and supervised more than 50 postdocs and 20 doctoral students in all major areas of Mathematical Logic as well as in the Foundations of Mathematics.

Dr Claudio Ternullo is a Beatriu de Pinós Post-Doctoral Fellow at the University of Barcelona (2020-present). He earned two PhDs in Philosophy at Pisa (2005) and Liverpool (2012). At Liverpool, he was also, for several years (2008-2011), a Temporary Lecturer of Logic. Between 2013 and 2015, he was a John Templeton Post-Doctoral Fellow (Project ID \#35216: 'The Hyperuniverse. Laboratory of the Infinite') at the University of Vienna's KGRC under the supervision of Prof Sy-David Friedman. His research has fundamentally focussed on the foundations of set theory, in particular on the issue of whether, as a consequence of set-theoretic incompleteness, there are many, alternative versions of mathematical (set-theoretic) truth. His research interests also lie in mathematical platonism, new axioms, multiverse conceptions and the problem of justification in mathematics. He is the author of several specialised articles, and of a monograph ('The Hyperuniverse. Laboratory of the Infinite'). 